\title{
Cytokine release in sepsis
}

\author{
Ian Burkovskiy $^{1 *}$, Joel Sardinha ${ }^{1 *}$, Juan Zhou ${ }^{2,3}$, Christian Lehmann ${ }^{1,2,3 \#}$ \\ ${ }^{1}$ Department of Pharmacology, Dalhousie University, Halifax, Canada \\ ${ }^{2}$ Department of Anesthesia, Dalhousie University, Halifax, Canada \\ ${ }^{3}$ Department of Microbiology \& Immunology, Dalhousie University, Halifax, Canada \\ Email: "hlehmann@dal.ca
}

Received 1 June 2013; revised 1 July 2013; accepted 1 August 2013

Copyright (c) 2013 Ian Burkovskiy et al. This is an open access article distributed under the Creative Commons Attribution License, which permits unrestricted use, distribution, and reproduction in any medium, provided the original work is properly cited.

\begin{abstract}
Despite the advances in the therapeutic approaches, health care protocols and policies, sepsis continues to be an important problem in clinical medicine. High lethality of sepsis cases calls for a detailed and critically important study of the pathophysiology of sepsis. In this review, we discuss pathomechanisms of sepsis and the role of cytokines that are released in sepsis. We propose that the systemic levels of cytokines are not always reflecting the pathological picture and the immune status of the patient. One of the emerging approaches which may bring an effective treatment strategy exploits the endocannabinoid system for its immunomodulatory properties. Following from that, the research in this particular field is very important as it can bring understanding behind the complicated pathophysiology of sepsis.
\end{abstract}

Keywords: Sepsis; Cytokine; Inflammation; Immunology

\section{INTRODUCTION}

Sepsis is the systemic inflammatory response to an infection that is usually associated with tissue hypoperfusion and multi-organ dysfunction [1]. According to generally accepted definitions, there are three clinical stages of sepsis-sepsis, severe sepsis and septic shock. Each individual stage is based on the complexity and the severity of the symptoms. Prevalence of septic shock and severe sepsis in intensive care units and emergency rooms continues to be high, despite the advances in surgical critical care and techniques, therapeutic approaches, establishment of new health care protocols and policies and development of new drugs [2,3]. One of the fundamental pathological characteristics of sepsis is the inability to

${ }^{*}$ These authors contributed equally.

${ }^{\#}$ Corresponding author. maintain the balance between excessive and inadequate inflammation [4].

Sepsis is considered to be the 10th leading cause of death in the United States, with approximately a million severe sepsis cases each year in the United States and an estimated 18 million cases of sepsis globally [5,6]. Modern therapeutic approaches are more frequent in utilizing aggressive immunosuppressive and chemotherapeutic treatments that compromise normal defense mechanisms and contribute to the increase in sepsis case numbers [2]. In addition to being a critical health condition, severe sepsis also puts a heavy socio-economic strain on the health care system [7]. Literature reports that the care of patients with sepsis brings an annual cost of the care to $\$ 17$ billion, in the United States alone [5]. In Canada, a study conducted by Letarte et al. [7] investigated the costs of severe sepsis and septic shock in the province of Quebec. The study reported a cost estimate between \$36.4 and \$72.9 million per year and concluded that the cost of severe sepsis is a significant economical burden for the Quebec health care system [7]. Finally, the patients that survive severe sepsis have a substantial reduction in their overall quality of life $[8,9]$.

\section{ETIOLOGY AND PATHOMECHANISMS OF SEPSIS}

Etiologically, any infectious agent that enters the bloodstream of a patient and generates a systemic immune response can cause sepsis. However, the presence of the pathogen in the blood is not a requirement for the clinical symptoms of sepsis to manifest themselves-as the presence of pathogen's signaling molecules or inflammatory mediators, which are released into the circulation from the source of infection, is sufficient for sepsis induction [10]. While sepsis can potentially be caused by many infectious agents such as bacteria, fungi, parasites or virus [11], the majority of cases of sepsis are reported to be due to bacteria [10]. 
The detection of the bacteria that causes sepsis strongly depends on the severity of the clinical picture with bacteria being detected in approximately $20 \%$ to $40 \%$ of patients with severe sepsis and in $40 \%$ to $70 \%$ of cases with septic shock [12]. One European study reported that the most frequent infectious agent that was associated with sepsis was Escherichia coli, with $22.7 \%$ share of detected cases [13]. A more detailed study by Vincent et al. [14] surveyed the prevalence and outcomes of infection in intensive care units from 75 countries. The results revealed that the microbiological culture results were positive in $70 \%$ of the infected patients with $62 \%$ of the positive isolates being gram-negative organisms, $47 \%$ being gram-positive, and 19\% being fungal in [14].

The first step in host defense is the recognition of microorganisms via binding of pattern-recognition recaptors, such as the Toll-like receptor family (TLRs) to highly conserved molecules called pathogen-associated molecular proteins (PAMPs) [15]. TLRs then recruit adaptor proteins to the cell surface and the adaptor proteins, in turn, activate a series of cytoplasmic kinases, initiating cascades that activate various transcription factors [15]. Genes that are induced by these transcription factors prepare the cell to undergo one of the potential main responses-rapidly undergoing apoptosis if the cell is overwhelmingly infected, fighting the infection with pro-inflammatory molecules and proliferating or suppressing the inflammatory reaction if no pathogens are longer detected [15].

First responding cells are monocytes and macrophages, which through induction of early response genes such as TNF $\alpha$ and IL-6 rapidly produce inflammatory cytokines and chemokines, amplifying the inflammatory response [15]. This is followed by an activation of lymphocytes as an adaptive immune response, as well as the coordination of later phases of the immune response [15]. Additionally, nitric oxide (NO) that is produced by endothelial cells induces vasodilation and an increase in leukocyte delivery to the sites of immune-activity [15].

\section{ENDOCANNABINOID SYSTEM}

The endocannabionoid system has emerged as a potential therapeutic target in sepsis treatment due to its immune modulatory functions. This endogenous system derives its name from the plant Cannabis sativa which contains many phytocannabinoids that activate endocannabinoid receptors within our bodies. The two most well characterized endocannabinoid receptors are cannabinoid recaptor $1\left(\mathrm{CB}_{1}\right)$ and cannabinoid receptor $2\left(\mathrm{CB}_{2}\right)$. The $\mathrm{CB}_{1}$ receptors are found throughout our bodies, but are highly concentrated in our central nervous system where they are implicated in modulating neurotransmitter release. Alternatively, the $\mathrm{CB}_{2}$ receptors are mainly localized on the surface of our immune cells, thus implicating a role within our immune system. Exploiting the activity of these receptors may prove to be beneficial in sepsis treatment. It has been well established that activation of the $\mathrm{CB}_{2}$ receptors initiates immunosuppressive mechanisms. One component of this suppressive mechanism involves altering the cytokines released by immune cells. $\mathrm{CB}_{2}$ receptor activation results in a reduction of pro-inflammatory cytokine release from leukocytes as well as an increased secretion of immunosuppressive cytokines. This outcome may have a beneficial effect if initiated during a pro-inflammatory phase of the septic cascade. However, due to the variability of the immune state during septic progression, immunosuppressive therapeutics can be detrimental, leaving the patient vulnerable to infections. As a result, measurement of cytokine levels in the blood was suggested as a method for detecting the condition of the patient's immune system. A proper understanding of the patient's immune state during sepsis is vital for appropriate treatments.

\section{CYTOKINE RELEASE}

Cytokines are low molecular weight signaling molecules secreted by a variety of cells that are used for cellular communication. Cytokines exist as proteins, glycoproteins, or peptides, and have functional roles in immunomodulation as well as development. They are involved in cellular activation, trafficking, signaling events, proliferation, differentiation and migration [16]. Cytokines exert their effects on targets by binding to cell surface recaptors. Receptor activation subsequently leads to modulation of intracellular cascades, eventually causing a biological effect. A few cytokines are constitutively expresses, however a majority of cytokines is produced in response to antigens, other cytokines, or cellular stressors. Due to the facultative expression of these molecules, gene expression of cytokines is tightly regulated, with rapid transcription and translation of proteins on demand. As an example, activation of transcription factor nuclear factor $\kappa \mathrm{B}(\mathrm{NF}-\kappa \mathrm{B})$ causes the rapid production and release of pro-inflammatory cytokines such as IL-1 [16].

Cytokines exert their effects in a dose dependent manner and have relatively short half lives in the extracellular environment. Therefore, cytokine levels can fluctuate drastically in microenvironments during their short duration of action. Once cytokines are released, they can act on their targets in either an autocrine or paracrine fashion. Multiple cytokines can also interact in the extracellular environment, causing a plethora of different effects. All cytokines are pleiotropic, which indicates that they can have variable effects based on the receptors they bind to. Alternatively, many cytokines can be redundant because they can have the same functional outcome. Different 
cytokines can act in a synergistic manner, which would elevate the response to a level greater than the additive effects of the individual cytokines. In contrast, cytokines can also behave antagonistically, where the effects of some cytokines may be diminished by others. This flexibility, of multiple functional outcomes, equips the immune system to activate and coordinate entire networks of immune cells from a small number of cytokine producing cell types [17].

Cytokines can be categorized based on their functional response into Type I or T-helper I (Th1) and Type II or T-helper 2 (Th2). Th1 cytokines are responsible for infections that produce a cell mediated immune response, while Th2 cytokines produce an antibody mediated immune response [17]. Another method of categorizing cytokines is based on their immune elicited outcome into either pro-inflammatory or anti-inflammatory cytokines (Table 1). Pro-inflammatory cytokines help up regulate the immune system when antigens are detected, or during cellular stressors. Anti-inflammatory cytokines help abate and modulate an up-regulated immune response bringing it back to homeostasis after the threat has been eliminated. The major pro-inflammatory cytokines discussed in this paper are TNF $\alpha$, IL- $1 \beta$, IL-6 and IL-17, while the main anti-inflammatory cytokines discussed will be IL-10, IL-4, IL-13 and TGF- $\beta$ (Table 1).

\section{PRO-INFLAMMATORY CYTOKINES}

Tumour necrosis factor alpha (TNF $\alpha)$ is a pro-inflammatory cytokine with a primary function to promote inflammation. This cytokine achieves this by signaling through specific receptors to induce gene expression of key inflammatory products. TNF $\alpha$ is shown to be a potent inducer of endothelial adhesion molecules to enable continuous recruitment of immune cells to help fight the infection [18]. TNF- $\alpha$ and IL-1 have also been demonstrated to act synergistically in order to initiate and promote inflammatory signalling pathways [19].

Interleukin-6 (IL-6) is classified as both a pro-inflammatory and anti-inflammatory cytokine and is linked with bacterial sepsis. Serum levels of IL-6 have been regarded as a surrogate marker for disease severity in sepsis. Moreover, IL-6 has also been shown to be an important mediator of fever and immuno-acute phase responses [18]. This particular cytokine was also extensively investigated in the past few years due to its involvement in differentiating the newly defined $\mathrm{T}$ helper 17 (TH17) subset of CD4 + T cells, among with TGF- $\beta$ and IL-21 [18]. In addition, IL-6 appears to be crucial to the production of functional tissue factor complexes [20].

Interleukin- 1 beta (IL- $1 \beta$ ) is a pro-inflammatory cytokine that is a potent inducer of endothelial adhesion molecules to enable further recruitment of immune cells,

Table 1. Anti-inflammatory/inflammtory cytokines and their functional role in sepsis.

\begin{tabular}{|c|c|}
\hline $\begin{array}{l}\text { Inflammatory } \\
\text { cytokines }\end{array}$ & Function in sepsis \\
\hline $\mathrm{TNF} \alpha$ & $\begin{array}{l}\text { Promotion of inflammation, potent inducer of endothelial adhesion molecules. May act synergistically with IL-1 to promote } \\
\text { and initiate inflammatory pathways. }\end{array}$ \\
\hline $\mathrm{IL}-1 \beta$ & $\begin{array}{l}\text { Potent inducer of endothelial adhesion molecules to enable recruitment of immune cells. Involved in promotion of } \\
\text { inflammatory signaling pathway, IL- } 1 \beta \text { can also activate the release of NO by both the endothelial and vascular smooth } \\
\text { muscle cells. }\end{array}$ \\
\hline IL-6 & $\begin{array}{l}\text { Important mediator of fever and immno-acute phase responses, involved in differentiating newly defined T helper 17 (TH17) } \\
\text { subset of CD4 + T cells. Also involved in production of functional tissue factor complexes. }\end{array}$ \\
\hline IL-17/IL-17A & $\begin{array}{l}\text { Involved in recruitment of monocytes and neutrophils to inflammation site, also expressed in natural killer (NK) cells. May } \\
\text { have indirect chemo-attractive properties due to the upregulation of granulocyte colony stimulaing factor (G-CSF) and CXC } \\
\text { chemokines. }\end{array}$ \\
\hline \multicolumn{2}{|c|}{$\begin{array}{l}\text { Anti-inflammatory } \\
\text { cytokines }\end{array}$} \\
\hline TGF- $\beta$ & $\begin{array}{l}\text { Modulates the activity of other cytokines through either enhancing or antagonizing effects. Can diminish the proliferation } \\
\text { and differentiation of T cells and B cells. Can also promote a state of resolution and repair. }\end{array}$ \\
\hline IL-4 & $\begin{array}{l}\text { Not released systemically into the bloostream during sepsis. IL-4 suppresses macrophage activity and has general } \\
\text { immunosuppressive effects. }\end{array}$ \\
\hline IL-10 & $\begin{array}{l}\text { Involved in modulation of the pro-inflammatory response, serves to move the immune system from a cell mediated response } \\
\text { to a humoral response. Blocks the innate immune response. Can also indirectly block pro-inflammatory cytokine activity. }\end{array}$ \\
\hline IL-13 & $\begin{array}{l}\text { Affects cell surface expression of different receptors in macrophages and monocytes. Down regulates CD-14 receptor } \\
\text { expression, also down regulates the expression of many pro-inflammatory cytokines such as TNF } \alpha \text { \& IL-1 in monocytes. }\end{array}$ \\
\hline
\end{tabular}


involved in promotion of inflammatory signaling pathways [18]. IL- $1 \beta$ can also activate the release of NO by both the endothelial and vascular smooth muscle cells, via increased transcription and activity of the inducible form of NO synthase [18].

Interleukin-17 or Interleukin-17A (IL-17/IL-17A) is a pro-inflammatory cytokine that is produced by $\mathrm{T}$ helper 17 , a subset of CD4 + T cells. IL-17 is involved in recruitment of monocytes and neutrophils to inflammation site [18]. IL-17 is also expressed in natural killer (NK) cells and this pro-inflammatory cytokine has been shown to be detrimental in the survival outcome of common lymphoid progenitor (CLP), the murine model of polymicrobial sepsis [21]. Literature reports that IL-17A may also be considered to have indirect chemoattractive properties due to the up-regulation of granulocyte colony-stimulating factor (G-CSF) and CXC chemokines, resulting in enhanced recruitment of neutrophils to the site of infection, resulting in an efficient bacterial clearance [22].

\section{ANTI-INFLAMMATORY CYTOKINES}

One of the main anti-inflammatory cytokines is IL-10. IL-10 helps modulate the pro-inflammatory response during early stages of sepsis by limiting exaggerated proinflammatory effects as well as making targets more tolerant to repeated pro-inflammatory stimuli [15]. However, in later stages, more sustained levels of IL-10 serve to move the immune system from a cell mediated response (Th1; innate immunity) to a humoral response (Th2; adaptive immunity). Known cellular sources of IL-10 include macrophages, dendritic cells, B-lymphocytes, T-regulatory cells (TREGS), and natural killer $\mathrm{T}$ cells (NKT cells) [23]. IL-10 blocks the innate immune response by inhibiting development and cytokine release by Th1 cells, as well as blocking production of certain pro-inflammatory cytokines. Apart from blocking proinflammatory cytokine release, IL-10 can also indirectly block pro-inflammatory cytokine activity by inducing the expression of soluble antagonistic receptors such as IL-1 receptor antagonist (IL-1RA) and soluble TNF receptors (sTNFR). These soluble antagonistic receptors bind to the pro-inflammatory cytokines and prevent them from activating their targets, effectively diminishing the extent of pro-inflammatory stimulation. The role of IL-10 in sepsis pathogenesis is not well understood. Conflicting results have been shown by different groups using IL-10 in survival outcome studies [24]. One study pretreated mice with anti-IL-10 antibody before inducing a septic state and found a decreased ability to survive in comparison to controls [25]. Conversely, another study tested mice lacking the IL-10 gene and observed survival when anti-IL-10 antibodies were administered at different time points [26]. These IL-10 deficient mice showed improved survival only if anti-IL-10 antibodies were given after a delay of initiating the septic challenge, presumably following the initial pro-inflammatory cascade, but not when anti-IL-10 antibodies were administered directly after the septic challenge. The results of these, as well as many other studies, indicate that the role of IL-10 in sepsis may be dependent of immune state of the patient, and effective treatments involving this cytokine are time critical during sepsis pathogenesis [23].

Interleukin 4 (IL-4) is another cytokine in the immune system arsenal that has immunosuppressive effects. This cytokine is unique because during sepsis it is not released systemically into the bloodstream. Studies have not been able to detect changes in IL-4 plasma levels of septic patients, however isolated splenocytes from septic mice did secrete higher levels of this cytokine when stimulated ex-vivo [23]. These results suggest a local effect of IL-4 immune suppression during a septic state, limiting its therapeutic potential. IL-4 works by inhibiting Th1 cells from polarizing and differentiating, as well as playing an important role in B cell differentiation, thereby promoting a Th2 mediated response [23]. IL-4 suppresses macrophage activity by preventing their cytotoxic activity, parasite killing, and nitric oxide production $[27,28]$.

Interleukin 13 (IL-13) shares many similar characteristics to IL-4, from its gene location (close proximity to IL-4 gene) to the receptor it activates (IL-4 type I recaptor) [27]. However, unlike IL-4, IL-13 has negligible effects on Th2 cell function. IL-13 has been shown to affect cell surface expression of different receptors in macrophages and monocytes, such as up regulating the expression of some integrins, while leaving other cellcell interacting receptors unaffected [29]. An important mechanism by which IL-13 exerts its anti-inflammatory properties is through the down regulation of CD-14 receptor expression. CD-14 is an important co-receptor with Toll Like Receptor 4 (TLR4) that recognises lipopolysaccharide (LPS) and elicits a strong immune response [29]. Furthermore, IL-13 also down regulates the expression of many pro-inflammatory cytokines such as TNF $\alpha$ \& IL-1 in monocytes [27]. Investigations into the role of IL-13 during sepsis indicate that mice show elevated levels of IL-13, and anti-IL-13 antibody treatment reduces their survival [27]. Interestingly, the anti-IL-13 antibody treated mice showed an increased neutrophil influx into tissues, but no effect on bacterial load and other leukocyte infiltration. The excess neutrophil influx into tissues showed an increase in organ damage indicating progression of the septic pathophysiology [27].

Transforming Growth Factor- $\beta$ (TGF- $\beta$ ) has many regulatory properties, therefore can have both pro and antiinflammatory properties. TGF- $\beta$ modulates the activity of other cytokines through either enhancing or antago- 
nizing effects [27]. TGF- $\beta$ can diminish the proliferation and differentiation of $\mathrm{T}$ cells and $\mathrm{B}$ cells by blocking production of specific cytokines like IL-2 [23]. Studies comparing cytokine levels showed elevated systemic levels of TGF- $\beta$ in trauma patients but no significant change in patients with septic shock [23]. Studies with TGF- $\beta$ knockout mice showed an exaggerated inflammatory reaction, indicating the anti-inflammatory properties of this cytokine [27]. Furthermore, TGF- $\beta$ can promote a state of resolution and repair, opposing the effects of a pro-inflammatory cascade similar to IL-4. These results indicate a more local response of TGF- $\beta$, similar to IL-4, limiting its therapeutic potential in systemic diseases like sepsis $[23,27]$.

\section{CONCLUSION}

The mortality of severe sepsis and septic shock ranges from $30 \%$ to $70 \%$ respectively. Clinically, there are only very few treatments shown to be beneficial in sepsis, e.g. antibiotics and vasopressors [15]. There are many potential explanations for little to no impact of the other therapies-such as the timing of the drug administration, systemic levels of cytokines not always reflecting the pathological picture and the immune status of the patient. Currently there are reports in literature that indicate a new potential focus on specific development of a clinically applicable therapy that would utilize the immunomodulating properties of the endocannabinoid system to combat the onset of sepsis [30,31]. The strategy of the approach would be revolving around the condition when the pro-inflammatory cytokine levels appear to be high. In summary, the research in this field is highly important in order to advance our understanding and to develop an effective therapeutic strategy.

\section{REFERENCES}

[1] Dellinger, R.P. (2008) Steroid therapy of septic shock: The decision is in the eye of the beholder. Critical Care Medicine, 36, 1987-1989.

doi:10.1097/CCM.0b013e31817d7ee4

[2] Balk, R.A. (2000) Severe sepsis and septic shock. Critical Care Clinics, 16, 179-192. doi:10.1016/S0749-0704(05)70106-8

[3] Wheeler, A.P. and Bernard, G.R. (1999) Treating patients with severe sepsis. New England Journal of Medicine, 340, 207-214. doi:10.1056/NEJM199901213400307

[4] Cohen, J. (2002) The immunopathogenesis of sepsis. $\mathrm{Na}$ ture, 420, 885-891. doi:10.1038/nature01326

[5] Angus, D.C., Linde-Zwirble, W.T., Lidicker, J., Clermont, G., Carcillo, J. and Pinsky, M.R. (2001) Epidemiology of severe sepsis in the United States: Analysis of incidence, outcome, and associated costs of care. Critical Care Medicine, 29, 1303-1310.

doi:10.1097/00003246-200107000-00002
[6] Global Sepsis Alliance (2010) International organizations declare sepsis a medical emergency: Issued by an expert panel representing 20 adult and pediatric intensive care societies.

[7] Letarte, J., Longo, C.J., Pelletier, J., Nabonne, B. and Fisher, H.N. (2002) Patient characteristics and costs of severe sepsis and septic shock in Quebec. Journal of Critical Care, 17, 39-49. doi:10.1053/jcrc.2002.33028

[8] Perl, T.M., Dvorak, L., Hwang, T. and Wenzel, R.P. (1995) Long-term survival and function after suspected gram-negative sepsis. JAMA, 274, 338-345. doi:10.1001/jama.1995.03530040066043

[9] Heyland, D.K., Hopman, W., Coo, H., Tranmer, J. and McColl, M.A. (2000) Long-term health-related quality of life in survivors of sepsis: Short Form-36: A valid and reliable measure of health-related quality of life. Critical Care Medicine, 28, 3599-3605. doi:10.1097/00003246-200011000-00006

[10] Holub, M. and Zavada, J. (2011) Clinical aspects of sepsis. Contributions to Microbiology, 17, 12-30.

[11] Bone, R.C., Balk, R.A., Cerra, F.B., Dellinger, R.P., Fein, A.M., Knaus, W.A., Schein, R.M. and Sibbald, W.J. (1992) Definitions for sepsis and organ failure and guidelines for the use of innovative therapies in sepsis. The ACCP/SCCM Consensus Conference Committee. American College of Chest Physicians/Society of Critical Care Medicine. Chest, 101, 1644-1655. doi:10.1378/chest.101.6.1644

[12] Brun-Buisson, C., Doyon, F., Carlet, J., Dellamonica, P., Gouin, F., Lepoutre, A., Mercier, J.C., Offenstadt, G. and Régnier, B. (1995) Incidence, risk factors, and outcome of severe sepsis and septic shock in adults: A multi-center prospective study in intensive care units. French ICU Group for Severe Sepsis. JAMA, 274, 968-974. doi:10.1001/jama.1995.03530120060042

[13] Luzzaro, F., Viganò, E.F., Fossati, D., Grossi, A., Sala, A., Sturla, C., Saudelli, M. and Toniolo, A. (2002) AMCLI Lombardia Hospital Infectious Study Group: Prevalence and drug susceptibility of pathogens causing bloodstream infections in northern Italy: A two-year study in 16 hospitals. European Journal of Clinical Microbiology \& Infectious Diseases, 21, 849-855.

[14] Vincent, J.L., Rello, J., Marshall, J., Silva, E., Anzueto, A., Martin, C.D., Moreno, R., Lipman, J., Gomersall, C., Sakr, Y., et al. (2009) EPIC II Group of Investigators: International study of the prevalence and outcomes of infection in intensive care units. JAMA, 302, 2323-2329. doi:10.1001/jama.2009.1754

[15] Russel, J.A., Boyd, J., Nakada, T., Thair, S. and Walley, K.R. (2011) Molecular mechanisms of sepsis. Contributions to Microbiology, 17, 48-85.

[16] Klaassen, C. D. and Watkins, J. B. (1999) Casarett and Doull's toxicology: The basic science of poisons. McGrawHill, New York.

[17] Whiteside, T. L. (2007). Introduction to cytokines as targets for immunomodulation. In Cytokines in Human Health, Humana Press, 1-15. doi:10.1007/978-1-59745-350-9 1

[18] Chong, D.L.W. and Sriskandan, S. (2011) Pro-inflamma- 
tory mechanisms in sepsis. Contributions to Microbiology, 17, 86-107.

[19] Dinarello, C.A. (2000) Proinflammatory cytokines. Chest, 111, 503-508. doi:10.1378/chest.118.2.503

[20] Riewald, M. and Ruf, W. (2003) Role of coagulation protease cascades in sepsis. Critical Care, 7, 123-129. doi:10.1186/cc1825

[21] Flierl, M.A., Rittirsch, D., Gao, H., Hoesel, L.M., Nadeau, B.A., Day, D.E., Zetoune, F.S., Sarma, J.V., Huber-Lang, M.S., Ferrara, J.L. and Ward, P.A. (2008) Adverse functions of IL-17A in experimental sepsis. FASEB Journal, 22, 2198-2205. doi:10.1096/fj.07-105221

[22] Ye, P., Rodriguez, F.H., Kanaly, S., Stocking, K.L., Schurr, J., Schwarzenberger, P., Oliver, P., Huang, W., Zhang, P., Zhang, J., Shellito, J.E., Bagby, G.J., Nelson, S., Charrier, K., Peschon, J.J. and Kolls, J.K. (2001) Requirement of interleukin 17 receptor signaling for lung CXC chemokine and granulocyte colony-stimulating factor expression, neutrophil recruitment, and host defense. Journal of Experimental Medicine, 194, 519-527. doi:10.1084/jem.194.4.519

[23] Shubin, N.J., Monaghan, S.F. and Ayala, A. (2011). Antiinflammatory mechanisms of sepsis. Contributions to Microbiology, 17, 108-124. doi:10.1159/000324024

[24] Perl, M., Chung, C.S., Garber, M., Huang, X. and Ayala, A. (2006) Contribution of anti-inflammatory/immune suppressive processes to the pathology of sepsis. Frontiers in Bioscience, 11, 272-299. doi:10.2741/1797

[25] Hogaboam, C.M., Steinhauser, M.L., Schock, H., Lukacs, N., Strieter, R.M., Standiford, T. and Kunkel, S.L. (1998) Therapeutic effects of nitric oxide inhibition during experimental fecal peritonitis: Role of interleukin-10 and monocyte chemoattractant protein 1. Infection and Immunity, 66, 650-655.

[26] Song, G.Y., Chung, C.S., Chaudry, I.H. and Ayala, A. (1999) What is the role of interleukin 10 in polymicrobial sepsis: Anti-inflammatory agent or immunosuppressant? Surgery, 126, 378-383. doi:10.1016/S0039-6060(99)70180-7

[27] Opal, S.M. and DePalo, V.A. (2000). Anti-inflammatory cytokines. Chest Journal, 117, 1162-1172. doi:10.1378/chest.117.4.1162

[28] Vannier, E., Miller, L.C. and Dinarello, C.A. (1992) Coordinated antiinflammatory effects of interleukin 4: Interleukin 4 suppresses interleukin 1 production but upregulates gene expression and synthesis of interleukin 1 receptor antagonist. Proceedings of the National Academy of Sciences of the United States of America, 89, 4076-4080. doi:10.1073/pnas.89.9.4076

[29] de Waal Malefyt, R., Figdor, C.G., Huijbens, R., MohanPeterson, S., Bennett, B., Culpepper, J. and De Vries, J.E. (1993) Effects of IL-13 on phenotype, cytokine production, and cytotoxic function of human monocytes. Comparison with IL-4 and modulation by IFN-gamma or IL-10. The Journal of Immunology, 151, 6370-6381.

[30] Kianian, M., Al-Banna, N.A., Kelly, M. and Lehmann, C. (2013) Inhibition of endocannabinoid degradation in experimental endotoxemia reduces leukocyte adhesion and improves capillary perfusion in the gut. Journal of Basic and Clinical Physiology and Pharmacology, 24, 27-33. doi:10.1515/jbcpp-2012-0065

[31] Sardinha, J., Lehmann, C. and Kelly, M. (2013) Targeting the endocannabinoid system to treat sepsis. Signa Vitae, $\mathbf{8}$, 9-14. 